Pacific Journal of 


\title{
A THEORY OF NORMAL CURVES AND SOME APPLICATIONS
}

\author{
C. J. Titus
}

1. Introduction. The notion of the normal curve as introduced by Whitney [12] is important partly for the following reasons: the normal curves are dense in a natural sense in the regular $C^{\prime}$ curves; the combinatorial topology embodied in the self-intersection numbers is welldefined. The self-intersection numbers and another minor condition determine a curve up to a sense preserving homeomorphism of the plane onto itself. This fact follows, e.g., directly from work of Adkisson and MacLane [1] and Gehman [3] but will not be verified here since no direct use will be made. It is mentioned, however, since it clearly indicates the amount of information in the intersection numbers. This matter is discussed in detail and used in an essential way in [10].

In the literature, from the point of view of combinatorial topology, there has been, after the beginnings, an emphasis on the study of curves as point sets in their own right and suprisingly little on their study as mappings directly. In this paper relations between the intersections are defined and various properties of curves are described in terms of these relations. Some applications are then given primarily to clarify the sense in which the normal curves are dense among the regular curves and to show how one can obtain theorems on representations of curves directly which then imply theorems on the extensions of these representations to, e.g., interior mappings on the disk. For conditions on the intersection numbers that there exist an extension by an interior mapping, see [9] and [10].

Lemmas 1, 3, 4, 5, 7 are all implicity in the work of Whitney [12] and [13] and are proved here in more or less detail for completeness and in some cases because the direct development, in the special situation here, is more concise.

2. Basic concepts and techniques. A complex valued function $\zeta(t)$ defined on an interval of the real line is called a regular representation of a curve if it possesses a continuous non-vanishing derivative $\zeta^{\prime}(t)$. If, as will usually be the case, $\zeta$ has the period $2 \pi$ then $\zeta$ is a regular repesentation of a closed curve and the interval $[0,2 \pi)$ may be identified with the unit circle oriented by increasing $t$.

An image point $\zeta_{1}$ is called a simple crossing if there exist exactly two distinct numbers $t^{\prime}$ and $t^{\prime \prime}$ such that $\zeta\left(t^{\prime}\right)=\zeta\left(t^{\prime \prime}\right)=\zeta_{1}$ and such that

Received March 13, 1959. 
the tangent vectors $\zeta^{\prime}\left(t^{\prime}\right)$ and $\zeta^{\prime}\left(t^{\prime \prime}\right)$ are independent. A regular representation is called normal (Whitney [13], p. 281) if it has a finite number of simple crossing points and is one-to-one everywhere else.

Let $\zeta(t)=\zeta(t)+i \eta(t)$ be a normal representation of period $2 \pi$ on the real $t$-line and restricted to the interval $S=[0,2 \pi)$. Let $\left\{\zeta_{k}\right\}, k=$ $1,2, \cdots, n$, be the set of all simple crossing points. Let $\left\{t_{k}^{\prime}\right\},\left\{t_{k}^{\prime \prime}\right\}, k=$ $1,2, \cdots, n$, be the set of numbers in $S$ indexed so that $\zeta\left(t_{k}^{\prime}\right)=\zeta\left(t_{k}^{\prime \prime}\right)$, $t_{k}^{\prime}<t_{k}^{\prime \prime}$ and $0<t_{1}^{\prime}<t_{2}^{\prime}<\cdots<t_{n}^{\prime}<2 \pi$. From now on the simple crossing points $\zeta_{k}$ will be called vertices. Let the set of all numbers in $S$ which are mapped onto vertices by $\zeta$ be denoted also by $\left\{s_{k}\right\}, k=$ $1,2, \cdots, 2 n$, indexed so that $0<s_{1}<s_{2}<\cdots<s_{2 n}<2 \pi$. Given $s_{k}$ let $s_{k}^{*}$ be yet another name for the number in $\left\{s_{k}\right\}$ such that $\zeta\left(s_{k}\right)=\zeta\left(s_{k}^{*}\right)$. Let $\nu_{k}$ be given by

$$
-\nu_{k}=-\nu\left(s_{k}\right)=\operatorname{sgn}\left|\begin{array}{ll}
\xi^{\prime}\left(s_{k}\right) & \xi^{\prime}\left(s_{k}\right) \\
\eta^{\prime}\left(s_{k}^{*}\right) & \eta^{\prime}\left(s_{k}^{*}\right)
\end{array}\right|, k=1,2, \cdots, 2 n:
$$

and $\lambda_{k}$ by $\nu\left(t_{k}^{\prime}\right)$, i.e.,

$$
-\lambda_{k}=-\nu\left(t_{k}^{\prime}\right)=\operatorname{sgn}\left|\begin{array}{ll}
\xi^{\prime}\left(t_{k}^{\prime}\right) & \xi^{\prime}\left(t_{k}^{\prime}\right) \\
\eta^{\prime}\left(t_{k}^{\prime \prime}\right) & \eta^{\prime}\left(t_{k}^{\prime \prime}\right)
\end{array}\right|, k=1,2, \cdots, n .
$$

Given any pair of distinct vertices $\zeta_{j}$ and $\zeta_{k}$ exactly one of the following five relations $R$ holds:

$R 1 j \subset k$, i.e., $t_{k}^{\prime} \leqq t_{j}^{\prime}<t_{j}^{\prime \prime} \leqq t_{k}^{\prime \prime}$,

$R 2 j \supset k$, i.e., $t_{j}^{\prime} \leqq t_{k}^{\prime}<t_{k}^{\prime \prime} \leqq t_{j}^{\prime \prime}$,

$R 3 j$ links $k$ on the right, i.e., $t_{k}^{\prime}<t_{j}^{\prime}<t_{k}^{\prime \prime}<t_{j}^{\prime \prime}$, denoted by $j \in R_{k}$,

$R 4 j \operatorname{links} k$ on the left, i.e., $t_{j}^{\prime}<t_{k}^{\prime}<t_{j}^{\prime \prime}<t_{k}^{\prime \prime}$, denoted by $j \in L_{k}$,

$R 5 j$ and $k$ are disjoint, i.e., either $t_{j}^{\prime}<t_{j}^{\prime \prime}<t_{k}^{\prime}<t_{k}^{\prime \prime}$ or

$$
t_{k}^{\prime}<t_{k}^{\prime \prime}<t_{j}^{\prime}<t_{j}^{\prime \prime} \text {, denoted by } j \mid k \text {. }
$$

The sequence $\left\{s_{k}\right\}$, the function given by $\nu\left(s_{k}\right)$ and the star operation* determine what will be called the intersection sequence of the normal representation $\zeta$. A vertex $\zeta_{k}$ will be signed positive [negative] if $\nu\left(t_{k}^{\prime}\right)>0\left[\nu\left(t_{k}^{\prime}\right)<0\right]$. In a straight forward but mildly tedious way one can show that a knowledge of the signs of the vertices and the relations $R$ for each pair of vertices is equivalent to a knowledge of the intersection sequence.

Let $\tilde{\zeta}$ and $\zeta$ be a pair of regular representations defined on a common interval $I$. The following norm will be used:

$$
\| \zeta-\tilde{\zeta}||=\sup _{t \in I}\left\{|\zeta(t)-\tilde{\zeta}(t)|+\left|\zeta^{\prime}(t)-\tilde{\zeta}^{\prime}(t)\right|\right\} .
$$

LEMMA 1. The intersection sequence of a normal representation is stable; i.e., given any normal representation $\zeta$ on an interval $I$ there 
exists an $\varepsilon>0$ such that for every regular representation on $I$ for which $\|\zeta-\tilde{\zeta}\|<\varepsilon$, it follows that $\tilde{\zeta}$ is normal and possesses the same intersection sequence as $\zeta$; namely, $\operatorname{sgn} \zeta_{k}=\operatorname{sgn} \tilde{\zeta}_{k}$ for all $k$ and $\zeta_{k}$ and $\zeta_{j}$ satisfy the same relation $R$ as $\tilde{\zeta}_{k}$ and $\tilde{\zeta}_{j}$ for all $j$ and $k$.

Proof. Let $\gamma_{j}(t)=\alpha_{j}(t)+i \beta_{j}(t)$ and $\tilde{\gamma}_{j}(t)=\tilde{\alpha}_{j}(t)+i \check{\beta}_{j}(t)$ be a pair of regular representations defined on an interval $I_{j}$. To prove the lemma the following two facts (i) and (ii) will be used. Each follows in a known way; see e.g., Whitney, [13], p. 281, proof of Lemma 2.

(i) If $\gamma_{j}(t)$ and $\gamma_{k}(t)$ have no image points in common then there exists and $\varepsilon>0$ such that, whenever $\left\|\gamma_{j}-\tilde{\gamma}_{j}\right\|$ and $\left\|\gamma_{k}-\tilde{\gamma}_{k}\right\|$ are each less than $\varepsilon, \tilde{\gamma}_{j}(t)$ and $\tilde{\gamma}_{k}(t)$ have no image points in common.

(ii) If $\gamma_{j}(t)$ and $\gamma_{k}(t)$ have exactly one point in common, say $\gamma_{k}\left(t_{j}\right)=$ $\gamma_{k}\left(t_{k}\right)$, and if the tangent vectors $\gamma_{j}^{\prime}\left(t_{j}\right)$ and $\gamma_{k}^{\prime}\left(t_{k}\right)$ are independent then there exists and $\varepsilon>0$ such that, whenever $\left\|\gamma_{j}-\tilde{\gamma}_{j}\right\|$ and $\left\|\gamma_{k}-\tilde{\gamma}_{k}\right\|$ are each less than $\varepsilon, \tilde{\gamma}_{j}$ and $\tilde{\gamma}_{k}$ have exactly one point in common, say $\tilde{\gamma}_{j}\left(\tilde{t}_{j}\right)=\tilde{\gamma}_{k}\left(\tilde{t}_{k}\right)$; the tangents $\tilde{\gamma}_{j}^{\prime}\left(\tilde{t}_{j}\right)$ and $\tilde{\gamma}_{k}^{\prime}\left(\tilde{t}_{k}\right)$ are independent; and

$$
\operatorname{sgn}\left|\begin{array}{cc}
\tilde{\alpha}_{j}^{\prime}\left(\tilde{t}_{j}\right) & \tilde{\beta}_{j}^{\prime}\left(\tilde{t}_{j}\right) \\
\tilde{\alpha}_{k}^{\prime}\left(\tilde{t}_{k}\right) & \tilde{\beta}_{k}^{\prime}\left(\tilde{t}_{k}\right)
\end{array}\right|=\operatorname{sgn}\left|\begin{array}{ll}
\alpha_{j}^{\prime}\left(t_{j}\right) & \beta_{j}^{\prime}\left(t_{j}\right) \\
\alpha_{k}^{\prime}\left(t_{k}\right) & \beta_{k}^{\prime}\left(t_{k}\right)
\end{array}\right|
$$

Select numbers $\sigma_{k}$ so that $0=\sigma_{0}<s_{1}<\sigma_{1}<s_{2}<\cdots<s_{2 n}<\sigma_{2 n}=2 \pi$ and define the intervals $I_{j}=\left(\sigma_{j-1}, \sigma_{j}\right), j=1,2, \cdots, 2 n$. Let $\gamma_{j}(t)=\zeta \mid I_{j}$; i.e., $\gamma_{j}(t)=\zeta(t)$ on $I_{j}$. Now, given any distinct pair of representations $\gamma_{j}$ and $\gamma_{k}$ either (i) or (ii) applies. The smallest $\varepsilon$ associated with a distinct pair of representations is chosen and the lemma follows.

Let $[\zeta]$ denote the point set consisting of the images of $\zeta$.

LEMMA 2. Let $\zeta$ be a normal representation of a closed curve $(\zeta(t)$ has period $2 \pi)$ and let $\gamma(u)=\alpha(u)+i \beta(u)$ be a normal representa-

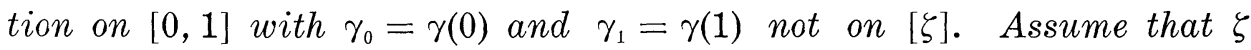
and $\gamma$ intersect one another in a finite number of points $\sigma_{\sigma}, \sigma=$ $1,2, \cdots, p$, which correspond to the parameter values $v_{\sigma}$ and $u_{\sigma}$ respectively; that the points $\zeta\left(v_{\sigma}\right)$ and $\zeta\left(u_{\sigma}\right)$ are not self intersection points of $\zeta$ and $\gamma$ respectively; that the tangents $\zeta^{\prime}\left(v_{\sigma}\right)$ and $\gamma^{\prime}\left(u_{\sigma}\right)$ are independent. Let $\omega_{0}\left[\omega_{1}\right]$ denote the index (winding number) of the curve $\zeta$ about the point $\gamma_{0}\left[\gamma_{1}\right]$. Then,

$$
\omega_{1}-\omega_{0}=\sum_{\sigma=1}^{p} \operatorname{sgn}\left|\begin{array}{ll}
\xi^{\prime}\left(v_{\sigma}\right) & \eta^{\prime}\left(v_{\sigma}\right) \\
\alpha^{\prime}\left(u_{\sigma}\right) & \beta^{\prime}\left(u_{\sigma}\right)
\end{array}\right| .
$$

Proof. Let the self intersection points of $\gamma$ correspond to parameter values $\left\{w_{\sigma}\right\}$ where $0<w_{1}<w_{2}<\cdots<w_{m}<1$. Let $\left\{w_{\sigma}^{\prime}\right\}$ be chosen so that $0=w_{0}^{\prime}<w_{1}<w_{1}^{\prime}<\cdots<w_{m}<w_{m}^{\prime}=1$ and so that $\gamma\left(w_{\sigma}^{\prime}\right)$ never 
lies on $[\zeta]$. Let $I_{j}$ denote the interval $\left[w_{j-1}^{\prime}, w_{j}^{\prime}\right], j=1,2, \cdots, m$. Let $\tilde{\omega}_{\sigma}$ denote the index of $\zeta$ about the point $\zeta\left(w_{\sigma}^{\prime}\right)$. Then, since $\gamma \mid I_{j}$ is an arc, one has by classical intersection theory

$$
\tilde{\omega}_{j+1}-\tilde{\omega}_{j}=\sum_{w_{j}^{\prime}<j \sigma^{<m_{j+1}}} \operatorname{sgn}\left|\begin{array}{ll}
\xi^{\prime}\left(v_{\sigma}\right) & \eta^{\prime}\left(v_{\sigma}\right) \\
\alpha^{\prime}\left(u_{\sigma}\right) & \beta^{\prime}\left(u_{\sigma}\right)
\end{array}\right| .
$$

Thus, since $\tilde{\omega}_{0}=\omega_{0}$ and $\tilde{\omega}_{m}=\omega_{1}$, one has

$$
\omega_{1}-\omega_{0}=\sum_{j=1}^{m}\left(\tilde{\omega}_{j}-\tilde{\omega}_{j-1}\right)=\sum_{\sigma=1}^{p} \operatorname{sgn}\left|\begin{array}{ll}
\xi^{\prime}\left(v_{\sigma}\right) & \eta^{\prime}\left(v_{\sigma}\right) \\
\alpha^{\prime}\left(u_{\sigma}\right) & \beta^{\prime}\left(u_{\sigma}\right)
\end{array}\right|
$$

as was to be shown.

Lemma 3. (Whitney, [13], Theorem 2, p. 281).

Let $\zeta$ be a normal representation with $\zeta_{0}=\zeta(0)$ on the outer bounda$r y$ of $[\zeta]$. (Then in every neighborhood of $\zeta_{0}$ there exist point about which $\zeta$ has index \pm 1 .) Then, the tangent winding number $\tau(\zeta)$ is given by

$$
\tau(\tau)=\sum_{\sigma=1}^{p} \lambda_{\sigma} \pm 1
$$

where the sign is chosen \pm as the sign of the non-zero index of about points chose to $\zeta_{0}$ is \pm .

Proof. This lemma is easily reduced to the Theorem of Whitney mentioned.

Lemma 4. Given a regular periodic representaticn $\zeta$ and an $\varepsilon>0$ there exists a regular periodic representation $\zeta$ such that

(i) $\|\zeta-\tilde{\zeta}\|<\varepsilon$ and there exists a $\delta>0$ such that for all $t$

(ii) $\{\zeta(\tau) \mid t-\delta<\tau<t+\delta\} \cap\{\tilde{\zeta}(\tau) \mid t-\delta<\tau<t+\delta\}$

is empty.

Proof. Let $\zeta^{*}(t) \equiv \frac{1}{2 h} \int_{t-h}^{t+h} \zeta(\tau) d \tau$ and define

$$
\tilde{\zeta}(t) \equiv \zeta(t)-i \rho\left[\zeta^{*}(t)\right]^{\prime} .
$$

The lemma will be proved by selecting $\tilde{\zeta}(t)$ to be of this form. For part (i), note that $\|\zeta-\tilde{\zeta}\|=\rho \max \left|\left(\zeta^{*}\right)^{\prime}\right|+\left|\left(\zeta^{*}\right)^{\prime \prime}\right|$. Since $\left(\zeta^{*}\right)^{\prime}$ and $\left(\zeta^{*}\right)^{\prime \prime}$ are continuous periodic functions and therefore bounded one can select a $\rho_{0}$ so that $\|\zeta-\tilde{\zeta}\|<\varepsilon$ for $\rho<\rho_{0}$. For part (ii) consider $|\zeta-\tilde{\zeta}|=\rho\left|\left(\zeta^{*}\right)^{\prime}\right|$. Since $\left(\zeta^{*}\right)^{\prime}$ converges uniformly to $\zeta^{\prime}$ as $h$ tends to zero and since $\left|\zeta^{\prime}\right|$ is bounded away from zero there exists an $h$ small enough that $\left|\left(\zeta^{*}\right)^{\prime}\right|=|\zeta-\tilde{\zeta}|$ is bounded away from zero. There exists 
therefore a $\delta>0$ so that $\left|\tilde{\zeta}\left(t^{\prime}\right)-\zeta\left(t^{\prime \prime}\right)\right|>0$ whenever $t-\delta<t^{\prime}$, $t^{\prime \prime}<t+\delta$.

Finally, to show that $\tilde{\zeta}$ can be chosen to be a regular representation, consider $\tilde{\zeta}^{\prime}=\zeta^{\prime}-i \rho\left(\zeta^{*}\right)^{\prime}$. Since $\left|\zeta^{\prime}\right|$ is bounded away from zero and $\left|\left(\zeta^{*}\right)^{\prime}\right|$ is bounded there is a $\rho$ small enough so that $\rho<\rho_{0}$ and $\tilde{\zeta}$ is never zero.

The idea of the intersection sequence has already been introduced. However, one can easily construct simple examples of intersection sequences which cannot come from a closed curve. For example, the sequence $t_{1}^{\prime}<t_{2}^{\prime}<t_{1}^{\prime \prime}<t_{2}^{\prime \prime}$, no matter what function $\nu\left(t_{\sigma}^{\prime}\right)$ is chosen cannot correspond to a closed curve in the plane. It does however correspond to a closed curve on the torus. See v. Sz. Nagy [7], p. 579. Thus these questions are apparently related to the homology properties of the imbedding manifold. The next theorem extends the results of Nagy to intersection sequences of closed curves in the plane. Denote the interval $t_{k}^{\prime} \leqq t \leqq t_{k}^{\prime \prime}$ by $T_{k}$ and let $\zeta \mid T_{k}$ denote the representation given by $\zeta$ restricted to the interval $T_{k}$.

THEOREM 1. Let $\zeta$ be a normal representation of a closed curve with $\zeta_{0}$ chosen on the outer boundary. Let $\omega_{k}$ be the index of the curve given by $\zeta \mid T_{k}$ about the point $\zeta(v)$ where $s_{j}=t_{k}^{\prime}>v>s_{j-1}, s_{0}=0$. Then

$$
\sum_{\sigma \in L_{k}} \lambda_{\sigma}=\sum_{\sigma \in R_{k}} \lambda_{\sigma}=\omega_{k} .
$$

Proof. The proof follows immediately by use of Lemma 2 and the section of $\gamma(t)$ as $\gamma(t) \equiv \zeta(t), 0 \leqq t \leqq v$. This gives

$$
\omega_{k}=\sum_{\sigma \in L_{k}} \lambda_{\sigma}
$$

The fact that

$$
\omega_{k}=\sum_{\sigma \in R_{k}} \lambda_{\sigma}
$$

follows similarly by choosing $\gamma(t) \equiv \zeta(t), 0 \leqq t \leqq w, s_{i}=t_{k}^{\prime \prime}<w<s_{i+1}$, and using $\nu\left(t_{\sigma}^{\prime}\right)=-\nu\left(t_{\sigma}^{\prime \prime}\right)$.

CoRollary 1. If $\zeta \mid T_{k}$ is a representation with $\zeta(u), t_{k}^{\prime}=s_{j}>u>s_{j-1}$, such that $\zeta(u)$ is in unbounded component of complement of $\left[\zeta \mid T_{k}\right]$, then

$$
\sum_{\sigma \in L_{k}} \lambda_{\sigma}=\sum_{\sigma \in k_{k}^{\prime}} \lambda_{\sigma}=0 .
$$

Proof. Use Theorem 1 and the fact the $\omega_{k}=0$ in this case.

Corollary 2. (Gauss-Nagy [7], Theorem I, p. 580), 
The number of $s_{\sigma}$ between $s_{j}$ and $s_{j}^{*}$ for any normal closed curve $\zeta$ is always even.

Proof. If $s_{\sigma}$ is between $t_{k}^{\prime}=s$, and $s_{j}^{*}=t_{k}^{\prime \prime}$ then the vertex corresponding to $s_{\sigma}$ either links $\zeta_{k}$ on the left, on the right or $\sigma \subset k$. Since $\sum_{\sigma \in L k} \lambda_{\sigma}=\sum_{\sigma \in R k} \lambda_{\sigma}$ the number of $s_{\sigma}$ between $t_{k}^{\prime}$ and $t_{k}^{\prime \prime}$, namely,

$$
\sum_{\sigma \in L_{k}}\left|\lambda_{\sigma}\right|+\sum_{\sigma \in L_{k}}\left|\lambda_{\sigma}\right|+2\left(\text { no. of } \zeta_{\sigma}, \sigma \subset k\right)
$$

is certainly even since $\sum_{\sigma \in L k}\left|\lambda_{\sigma}\right|$ and $\sum_{\sigma \in R k}\left|\lambda_{\sigma}\right|$ are both even or both odd.

THEOREM 2. Let $\tilde{\zeta}$ be a normal representation for which $\tilde{\zeta}_{0}=\tilde{\zeta}(0)$ is not a vertex. Let the indices with respect to $\tilde{\zeta}$ of points near $\tilde{\zeta}_{0}$ be denoted by $\tilde{\omega}_{0}^{+}$and $\widetilde{\omega}_{0}^{-}$where $\widetilde{\omega}_{0}^{+}=\widetilde{\omega}_{0}^{-}+1$. Then

$$
\tau(\tilde{\zeta})=\sum_{\sigma=1}^{n} \tilde{\lambda}_{\sigma}+\left(\tilde{\omega}_{0}^{+}+\tilde{\omega}_{0}^{-}\right)
$$

Proof. From the Whitney theorem, here Lemma 3, one has with $\zeta_{0}$ selected on the outer boundary that

$$
\tau(\zeta)=\sum_{\sigma=1}^{n} \lambda_{\sigma}+\left(\omega_{0}^{+}+\omega_{0}^{-}\right)
$$

The following identity follows directly from the definitions of the $\lambda_{\sigma}$, the relations $R$ and Theorem 1:

$$
\sum_{\sigma=1}^{n} \lambda_{\sigma}=\sum_{\sigma \mid k} \lambda_{\sigma}+\sum_{\sigma \succeq k} \lambda_{\sigma}+\sum_{\sigma \succeq k} \lambda_{\sigma}+2 \sum_{\sigma \in L_{k}} \lambda_{\sigma}-\lambda_{k} .
$$

Let $u$ be a number such that $\zeta(u)=\tilde{\zeta}(0)$ and $s_{j}=t_{k}^{\prime}<u<s_{j+1}$. Now, by use of Lemmas 1,2 and 4 one has, with $\zeta$ and $\tilde{\zeta}$ representing the same curve,

$$
\tilde{\omega}_{0}^{-}-\omega_{0}^{-}=\sum_{s_{1} \leqq s_{\sigma} \leqq t_{k}^{\prime}} \nu_{\sigma} ;
$$

but again from definitions of the $\lambda_{\sigma}$ and the relations $R$ one has

$$
\sum_{s_{1} \leq s_{\sigma} \leq t_{k}^{\prime}} \nu_{\sigma}=\sum_{\sigma \in L_{k}} \lambda_{\sigma}+\sum_{\sigma \subset k} \lambda_{\sigma} \text {. }
$$

It follows easily by use of Lemma 2 that

$$
\tilde{\omega}_{0}^{+}-\omega_{0}^{+}=\tilde{\omega}_{0}^{-}-\omega_{0}^{-} .
$$

Thus,

$$
\left(\tilde{\omega}_{0}^{+}+\tilde{\omega}_{0}^{-}\right)=2\left\{\sum_{\sigma \in L_{k}} \lambda_{\sigma}+\sum_{\sigma \supset k} \lambda_{\sigma}\right\}+\left(\omega_{0}^{+}+\omega_{0}^{-}\right) .
$$


Now, again from definitions of the $\lambda_{\sigma}$ and the relations $R$,

$$
\sum_{\sigma=1}^{n} \tilde{\lambda}_{\sigma}=\sum_{\sigma \mid k} \lambda_{\sigma}+\sum_{\sigma \subset k} \lambda_{\sigma}-\sum_{\sigma \supset k} \lambda_{\sigma}-\lambda_{k}
$$

Thus, by (D) and (E),

$$
\sum_{\sigma=1}^{n} \tilde{\lambda}_{\sigma}+\left(\tilde{\omega}_{0}^{+}+\tilde{\omega}_{0}^{-}\right)=\sum_{\sigma \mid k} \lambda_{\sigma}+\sum_{\sigma \subset k} \lambda_{\sigma}+\sum_{\sigma \supset k} \lambda_{\sigma}+2 \sum_{\sigma \in L_{k}} \lambda_{\sigma}-\lambda_{k}+\left(\omega_{0}^{+}+\omega_{0}^{-}\right) .
$$

But, using (C),

$$
\sum_{\sigma=1}^{n} \tilde{\lambda}_{\sigma}+\left(\tilde{\omega}_{0}^{+}+\tilde{\omega}_{0}^{-}\right)=\sum_{\sigma=1}^{n} \lambda_{\sigma}+\left(\omega_{0}^{+}+\omega_{0}^{-}\right)=\tau(\zeta),
$$

and the Theorem follows.

Let $\varphi_{k}$ denote the angle, $-\pi<\varphi_{k}<\pi$, measured from the direction of $\zeta^{\prime}\left(t_{k}^{\prime \prime}\right)$ to the direction of $\zeta^{\prime}\left(t_{k}^{\prime}\right)$. Let $\psi_{k}$ denote the change in the angle of $\zeta^{\prime}(t)$ as $t$ varies from $t_{k}^{\prime}$ to $t_{k}^{\prime \prime}$. Then, as usual, the concept of the tangent winding number can be extended to the closed curve represented by $\zeta \mid T_{k}$ by

$$
\tau\left(\zeta \mid T_{k}\right)=\frac{1}{2 \pi}\left(\psi_{k}+\varphi_{k}\right)
$$

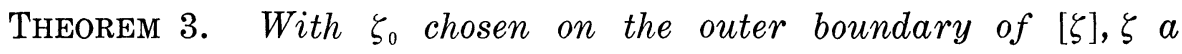
normal representation, then

$$
\tau\left(\zeta \mid T_{k}\right)=\sum_{\sigma \subset k} \lambda_{\sigma}+2 \sum_{\sigma \in L_{k}} \lambda_{\sigma}
$$

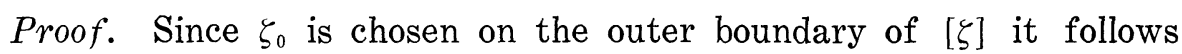
that the index of $\zeta \mid T_{k}$ about the point $\zeta_{0}$ is zero for all $k$. In order to compute $\tau\left(\zeta \mid T_{k}\right)$ Theorem 2 will be used and thus $\omega_{0}^{+} \mid T_{k}$ and $\omega_{0}^{-} \mid T_{k}$ must be computed. Explicitly, choose $u, t_{k}^{\prime}=s_{j}<u<s_{j+1}$, and let $\omega_{0}^{+} \mid T_{k}$ and $\omega_{0}^{-} \mid T_{k}$ be the indices of $\zeta \mid T_{k}$ about points near $\zeta(u)$ with as before $\omega_{0}^{+}\left|T_{k}=\omega_{0}^{-}\right| T_{k}+1$. Some computation using Lemmas 1 and 4 as in proof of Theorem 2 gives

$$
\omega_{0}^{+}\left|T_{k}+\omega_{0}^{-}\right| T_{k}=2 \sum_{\sigma \in L_{k}} \lambda_{\sigma}+\lambda_{\kappa} .
$$

By Theorem 2, as applied to $\zeta \mid T_{k}$,

$$
\tau\left(\zeta \mid T_{k}\right)=\sum_{\sigma \subset k} \lambda_{\sigma}+2 \sum_{\sigma \in L_{k}} \lambda_{\sigma}=\sum_{\substack{\sigma \subset k \\ \sigma \neq k}} \zeta_{\sigma}+\omega_{0}^{+}\left|T_{k}+\omega_{0}^{-}\right| T_{k}
$$

as was to be shown.

A regular closed curve is said to be of non-negative circulation, Loewner [6], p. 316, if the index of $\zeta$ about any point not on $[\zeta]$ is non-negative. Part of the importance of this concept comes from the 
following fact: Let $w=w(z)$ be an analytic function on $|z|<1$ and continuous on $|z| \leqq 1$. Then, the curve represented by $\zeta(t) \equiv w\left(e^{i t}\right)$, $z=\rho e^{i t}$, is of non-negative circulation. The proof of this statement follows easily from a lemma of Loewner, [6], Lemma 2, page 318, and is left to the interested reader.

THeORem 4. A closed curve given by a normal representation $\zeta$, with $\zeta_{0}$ on the outer boundary, is of non-negative circulation if and only if

$$
\sum_{\sigma \supset k} \lambda_{\sigma}+\sum_{\sigma \in R_{k}} \lambda_{\sigma} \geqq 0 \text { for all } k,
$$

and $\omega_{0}^{+}+\omega_{0}^{-}=1$. (As usual, $\omega_{0}^{+}$and $\omega_{0}^{-}$are the indices of $\zeta(t)$ about points near $\zeta_{0}$ ).

Proof. The condition that $\omega_{0}^{+}+\omega_{0}^{-}=1$ is clearly necessary. Consider $\zeta(v)$ with $s_{j}<v<s_{j+1}$. Let $\omega_{v}^{-}$denote the smaller of the indices of $\zeta$ about points near $\zeta(v)$. By Lemmas 1,2, and 4, again as in proof of Theorem 1 , one obtains, since $\omega_{0}^{-}=0$, that

$$
\omega_{v}^{-}=\sum_{s_{1} \leqq s_{\sigma} \leqq s_{j}} \nu\left(s_{\sigma}\right) .
$$

Now, $\omega_{v}^{-} \geqq 0$ for all $j$ since $\zeta$ is of non-negative circulation. Also it is clear that given any point $\pi$ not on $[\zeta]$ there is a $j$ such that the index of $\zeta$ about $\pi$ is either equal to $\omega_{v}^{-}$or to $\omega_{v}^{-}+1$. Thus, the condition

$$
\sum_{s_{1} \leqq s_{\sigma} \leqq s_{j}} \nu\left(s_{\sigma}\right) \geqq 0 \text { for all } j
$$

together with $\omega_{0}^{+}+\omega_{0}^{-}=1$ is necessary and sufficient. But given any $j$ there is an $k$, and vice-versa, such that

$$
\sum_{s_{1} \leq_{\sigma} \leqq s_{j}} \nu\left(s_{\sigma}\right)=\sum_{\sigma \subset k} \lambda_{\sigma}+\sum_{\sigma \in R_{k}} \lambda_{\sigma}
$$

For given $j$ choose $k$ to be the largest integer such that $t_{k}^{\prime} \leqq s_{j}$. Given $k$ choose $j$ to be the least integer such that $s_{j} \geqq t_{k}^{\prime}$. Using the fact that $\nu\left(t^{\prime}\right)=-\nu\left(t^{\prime \prime}\right)$ the above equality holds and the proof of Theorem 4 is complete.

A sub-set $Q$ of the vertices of a normal curve $\zeta$ will be called $e x$ terior provided that

$$
\sum_{\sigma \in L_{k}} \lambda_{\sigma}=0, \text { all } \zeta_{k} \varepsilon Q .
$$

Let $u$ be a number such that $t_{k}^{\prime}=s_{j}>u>s_{\jmath-1}$ and let $\hat{\omega}_{k}$ denote the index of $\zeta \mid T_{k}$ about the point $\zeta(u)$. Then as established in proof of Theorem 3 , one has for all $k$ that 


$$
\hat{\omega}_{k}=\sum_{\sigma \in L_{k}} \lambda_{\sigma}=\sum_{\sigma \in R_{k}} \lambda_{\sigma}
$$

Thus, $Q$ is exterior provided that

$$
\hat{\omega}_{k}=0, \quad \text { all } \zeta_{k} \in Q .
$$

There is a natural way to generate an exterior sub-set $Q$ of vertices to a given normal curve represented by $\zeta$ with $\zeta_{0}$ on the outer boundary

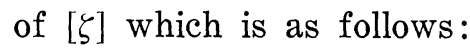

(i) $\zeta_{j_{1}}, \cdots, \zeta_{j_{m}} \in Q$ if $\hat{\omega}_{j_{\sigma}}$ are all zero, $j_{1}=1$ and $j_{\sigma}, \sigma>1$, is the smallest index such that $j_{\sigma}>j_{\sigma-1}$ and $j_{\sigma} \mid j_{\sigma-1}$;

(ii) $\zeta_{k_{1}}, \cdots, \zeta_{k_{m}} \in Q$ if $\hat{\omega}_{k_{\sigma}}$ are all zero and there exists a $\zeta_{j} \in Q$ such that $k_{1}$ is the smallest index with $k_{1} \subset j$ and $k_{\sigma}, \sigma>1$, is the smallest index with $k_{\sigma} \subset j, k_{\sigma}>k_{\sigma-1}$ and $k_{\sigma} \mid k_{\sigma-1}$.

Subsets $Q$ so generated will be called properly exterior.

THEOREM 5. Let $Q$ be a properly exterior sub-set of vertices of a normal representation $\zeta$ then

$$
\tau(\zeta)=\sum_{\sigma \in Q} \lambda_{\sigma}+\left(\omega_{0}^{+}+\omega_{0}^{-}\right)
$$

Proof. From the generation process it follows that each vertex $k \notin Q$ must link some vertex $j \in Q$ on the right; i.e., $k \in R_{j}$. Thus, to each vertex $k \notin Q$, one can associate a unique vertex $j \in Q$ by the following device: let $j \in Q$ be such that $k \in R$, and $j$ is minimal on $Q$ with respect to the order relation $R l$. Let $K_{j}$ be the set of vertices $k \notin Q$ which are associated by this device with the same vertex $j$. An argument, as in proof of Theorem 1, gives the fact that

$$
\sum_{\sigma \in K_{j} \cap R_{j}} \lambda_{\sigma}=0, \quad j \in Q .
$$

Thus,

$$
\sum_{\sigma=1}^{m} \lambda_{\sigma}=\sum_{\sigma \in Q} \lambda_{\sigma}+\sum_{j \in Q}\left(\sum_{\sigma \in K_{j} \cap R_{j}} \lambda_{\sigma}\right)=\sum_{\sigma \in Q} \lambda_{\sigma},
$$

and the proof follows by Theorem 2 .

\section{A criteria that a curve be simple.}

THeORem 6. A closed curve given by a normal representation $\zeta$, $\zeta_{0}$ on outer boundary, is simple and positively oriented if and only if

(i) $\tau(\zeta) \leqq 1$, i.e., tangent winding number not greater than 1 ,

(ii) $\tau\left(\zeta \mid T_{k}\right) \geqq 0$ for all $k$, i.e., tangent winding number of each subloop is non-negative, 
(iii) $\zeta$ is of non-negative circulation.

Proof. The conditions are clearly necessary for (ii) and (iii) follow immediately and (i) by the "Umlaufshtz", Hopf [4], p. 53.

From the theorems in $\S 2$ the hypotheses are equivalent, with $\zeta_{0}$ on the outer boundary, to

(i) $\sum_{\sigma=1}^{n} \lambda_{\sigma} \leqq 0$

(ii) $\sum_{\sigma \subset k} \lambda_{\sigma}+2 \sum_{\sigma \in L_{k}} \lambda_{\sigma} \geqq 0$ for all $k$,

(iii) $\sum_{\sigma \supset k} \lambda_{\sigma}+\sum_{\sigma \in L_{k}} \lambda_{\sigma} \geqq 0$ for all $k$.

By applying (iii), to a vertex $\zeta_{k}$ with $k \in Q, Q$ properly exterior and with $k$ maximal on $Q$, one has

$$
0 \leqq \lambda_{k}+\sum_{\sigma \in L_{k}} \lambda_{\sigma}=\lambda_{k}
$$

and thus that $\lambda_{k}=1$. Let $C_{k}$ denote the set of vertices in $Q$ covered by $k,(k$ covers $j$ if $k \neq j$ and if $k \supset \sigma \supset j$ implies $\sigma$ is either $k$ or $j$ ). Now, since $k \in Q$,

$$
\sum_{\sigma \subset k} \lambda_{\sigma}=\lambda_{k}+\sum_{j \in \sigma_{k}}\left(\sum_{\sigma \subset j} \lambda_{\sigma}\right)
$$

and by (ii)'

$$
\sum_{\sigma \subset j} \lambda_{\sigma} \geqq 0
$$

Thus

$$
\sum_{\sigma \subset k} \lambda_{\sigma} \geqq \lambda_{k}=1
$$

Finally, with $M$ denoting the maximal elements in $Q$, using Theorem 5,

$$
\tau-1=\sum_{\sigma=1}^{n} \lambda_{\sigma}=\sum_{k \in M}\left(\sum_{\sigma \subset k} \lambda_{\sigma}\right) \geqq(\text { no. of vertices in } M) \text {. }
$$

Thus, since $\tau-1 \leqq 0$, there are no vertices in $M$ and therefore $\zeta$ itself has no vertices. The proof is complete.

NotE. Actually the full strength of (ii) as well as (iii) were not used and the Theorem could be strengthened. However, in the hypotheses, (ii) and (iii) are convenient for various applications and it seems, at present, that estimations of the maximum index of $\zeta$ under (ii), (iii) with more general inequalities on $\tau$ are more interesting. Some estimates of this type will be published in a later paper. For an intuitive discussion of these kinds of "valence" estimates as applied to analytic functions see Umezawa [11]. 
4. Applications to regular curves. In order, among other things, to illustrate how these ideas for normal curves can be applied by a limiting process to regular curves the following theorem will be proved.

THeOREM 7. Let $\zeta$ be a regular representation of a closed curve which satisfies the following conditions:

(i) $\tau(\zeta) \leqq 1$.

Let the angle variation of $\zeta^{\prime}(t)$ on a sub-interval $T$ on the circle on which $\zeta$ is defined be denoted by $\Phi(T)$; thus $2 \pi \tau(\zeta)$ is the value of $\Phi$ on the whole circle.

(ii) $\Phi(T)>-\pi$ for all $T$.

(iii) There exists an $\varepsilon>0$ such that for all regular $\tilde{\zeta}$ with $\|\zeta-\tilde{\zeta}\|<\varepsilon$ it follows that $\zeta$ is of non-negative circulation.

Then $\zeta$ represents a simple positively oriented Jordan curve.

Some preliminary lemmas are required for the proof.

LEMma 5 (Whitney). Given a regular curve $\zeta$ there exist normal curves $\zeta^{*}$ such that $\left\|\zeta^{*}-\zeta\right\|$ is arbitrarily small.

Proof. See Whitney, [13], page 281, Lemma 2.

LeMma 6. Let $\zeta$ be a normal representation of a closed curve with $\Phi(T) \geqq-\pi$ on every interval $T$ on the circle then $\tau\left(\zeta \mid T_{k}\right) \geqq 0$ for all $k$.

Proof. From the geometric definition of $\tau\left(\zeta \mid T_{k}\right)$ one has, as in introduction to Theorem 3, that

$$
2 \pi \tau\left(\zeta \mid T_{k}\right)=\Phi\left(T_{k}\right)+\varphi_{k},-\pi<\varphi_{k}<\pi .
$$

Thus, $2 \pi \tau\left(\zeta \mid T_{k}\right)>-2 \pi$, and since $\tau\left(\zeta \mid T_{k}\right)$ is an integer the lemma follows.

LEMMA 7. If $\zeta$ is a regular representation which is not simple then there exist normal representations $\zeta^{*}$, with $\left\|\zeta-\zeta^{*}\right\|$ arbitrarily small, which are not simple.

Proof. If there exist distinct values $t^{\prime}$ and $t^{\prime \prime}$ such that $\zeta\left(t^{\prime}\right)=$ $\zeta\left(t^{\prime \prime}\right)$ then either the tangents $\zeta^{\prime}\left(t^{\prime}\right)$ and $\zeta^{\prime}\left(t^{\prime \prime}\right)$ are independent or they are not. If they are independent then, since the curve is regular, there exists a $\delta>0$ such that $\zeta$ is one-to-one on $\left[t^{\prime}-\delta, t^{\prime}+\delta\right]$ and also on $\left[t^{\prime \prime}-\delta, t^{\prime \prime}+\delta\right]$. Furthermore, with $\delta$ sufficiently small there arcs intersect only at $\zeta\left(t^{\prime}\right)=\zeta\left(t^{\prime \prime}\right)$. By the density lemma, Lemma 5 , $\zeta$ can be approximated arbitrarily closely by normal representation $\zeta^{*}$ and by the stability lemma, Lemma 1 , the intersection will be preserved.

If $\zeta^{\prime}\left(t^{\prime}\right)$ and $\zeta^{\prime}\left(t^{\prime \prime}\right)$ are not independent then it is clear that there is 
a regular curve arbitrarily close to $\zeta$ such that it intersects itself in at least one point with independent tangents and thus the method for the previous case can be applied. The proof is complete.

Proof of Theorem 7. By Lemma 5 there exist normal curves $\zeta^{*}$ such that $\left\|\zeta-\zeta^{*}\right\|$ is arbitrarily small. But when $\left\|\zeta-\zeta^{*}\right\|$ is small enough one has that $\tau\left(\zeta^{*}\right) \leqq 1$ and $\Phi(T)>-\pi$ on $\zeta^{*}$ since these conditions are easily seen to be stable. Furthermore, $\zeta^{*}$ is of non-negative circulation by the hypothesis (iii). Thus, by Theorem $6, \zeta^{*}$ is simple and positively oriented when $\left\|\zeta-\zeta^{*}\right\|$ is sufficiently small. But if $\zeta$ is not simple Lemma 7 implies that there exist normal $\zeta^{*}$, with $\left\|\zeta-\zeta^{*}\right\|$ arbitrarily small, which are not simple. This contradiction completes the proof.

5. Application to the theory of interior mappings. Regular representations which satisfy the condition $\Phi(T)>-\pi$ for all intervals $T$, are called close to convex, [5], p. 169.

The following theorem proved for interior mappings gives a result of Kaplan, [5], Theorem 1, p. 170, when the interior mapping is an analytic function. There is related work in these directions by Paatero [8], Umezawa [11] in the case of analytic functions. Their results depend strongly on the fact that the mappings are analytic and have no obvious analogue even when the interior mappings are solutions to elliptic systems of partial differential equations.

THEOREM 8. Let $\zeta$ be a regular representation of a close to convex curve with $\tau(\zeta)=1$ such that there exists a mapping $f$ continuous on $|z| \leqq 1, C^{\prime}$ and interior (=light and open) on $|z|<1$ with $f\left(e^{i t}\right) \equiv \zeta(t)$. Then the mapping $f$ is a homeomorphism.

Before beginning the proof consider the following lemmas.

Lemma 8. (Loewner). Let $f(z)$ be a mapping continuous on the annulus $0<\rho_{1} \leqq \rho \leqq \rho_{2}, z=\rho e^{i t}$, continuously differentiable and possessing a non-negative Jacobian on $\rho_{1}<\rho<\rho_{2}$. Then for every point $P$ not on $\left[f\left(\rho_{0} e^{i t}\right)\right] \cup\left[f\left(\rho_{2} e^{i t}\right)\right]$ the index of $f\left(\rho_{1} e^{i t}\right)$ about $P$ is not greater than the index of $f\left(\rho_{2} e^{i t}\right)$.

Proof. See Loewner, [6], Lemma 2, p. 318.

Lemma 9. If $f$ is a mapping continuous on $|z| \leqq 1, C^{\prime}$ and interior on $|z|<1$ and if $\zeta(t)=f\left(e^{i t}\right)$ is regular then there exists an $\varepsilon>0$ such that any regular $\tilde{\zeta}$, with $\|\zeta-\tilde{\zeta}\|<\varepsilon$, is of non-negative circulation. 
Proof. For any $k>0$ the curve $f\left((1-k) e^{i t}\right)$ is of non-negative circulation as is easily seen by Lemma 8 . As in the proof of Lemma 4 let

$$
\zeta^{*}=\frac{1}{2 h} \int_{t-h}^{t+h} \zeta(\tau) d \tau
$$

but let $\tilde{\zeta}=\zeta+i \rho\left(\zeta^{*}\right)^{\prime}$.

Consider $\tilde{\zeta}$ as a mapping from the annulus $1-k \leqq \rho \leqq 1$ in the $\rho t$-polar coordinate plane. The polar coordinate Jacobian is non-negative with $\rho$ and $k$ sufficiently small so that Lemma 8 again applies and one has that for every point $P$ in the plane, not on $[\zeta] \cup[\tilde{\zeta}]$, the index $\omega(p, \zeta)$ of $\zeta$ about $P$ is not less that $\omega(p, \tilde{\zeta})$;

$$
\omega(p, \zeta) \geqq \omega(p, \tilde{\zeta}) \text { for all } P \notin[\zeta] \cup[\tilde{\zeta}] \text {. }
$$

But given $k$ the $\rho$ can be chosen so small that, by the continuity of the degree functions on the space of regular representations,

$$
\omega(p, \tilde{\zeta}) \geqq \omega\left(P, f\left((1-k) e^{i t}\right)\right) \text { for all } P \notin[\tilde{\zeta}] \cup\left[f\left((1-k) e^{i t}\right]\right. \text {. }
$$

But with $\varepsilon$ chosen sufficiently small,

$$
\omega(p, \tilde{\zeta}) \geqq \omega(p, \tilde{\zeta}) \text { for all } P \notin[\tilde{\zeta}] \cup[\tilde{\zeta}] \text {. }
$$

Thus,

$$
\omega(p, \tilde{\zeta}) \geqq \omega(p, \tilde{\zeta}) \geqq \omega\left(p, f\left((1-k) e^{i t}\right)\right) \geqq 0
$$

for all $p \notin[\tilde{\zeta}] \cup[\tilde{\zeta}] \cup\left[f\left((1-k) e^{i t}\right)\right]$. Since none of the sets of image points involved contain any open sets it follows by a simple limiting process that

$$
\omega(p, \tilde{\zeta}) \geqq 0 \text { for all } p \notin[\tilde{\zeta}],
$$

or, in other words, that $\tilde{\zeta}$ is of non-negative circulation. The proof of the lemma is complete.

Proof of Theorem 8. By Lemma 9 the curve $\zeta$ satisfies the hypotheses of Theorem 7. Therefore, $\zeta$ represents a simple positively oriented Jordan curve. Therefore the mapping $f$ is one-to-one on the boundary and as is well known, even for general interior mappings (here, Lemma 8 easily applies) the mapping $f$ is a homeomorphism.

\section{BIBLIOGRAPHY}

1. V. W. Adkisson, and Saunders MacLane, Extensions of homeomorphisms on the sphere, Lectures in Topology, Univ. of Mich. Press (1941), 223-235.

2. C. F. Gauss, Werke (8), 272, 282-286.

3. H. M. Gehman On extending a continuous 1-1 correspondence of two plane continuous 
curves to a correspondence of their planes, Trans. Amer. Mth. Soc. 42 (1936), 79-81.

4. Heinz Hopf, Über die Drehung der Tangenten und Sehnen ebener Kurven, Compositio Math. 2 (1935), 50-62.

5. Wilfred Kaplan, Close to convex schlicht functions, Michgan Math. J. 1 (1952), 169-185.

6. Charles Loewner, A topological characterization of a class of integral operators, Annals of Math. 49 (1948), 316-332.

7. Julius v. Sz. Nagy, Über ein topologisches Problem von Gauss, Math. Zeit. 26 (1927), 579-592.

8. V. Paatero, Über die konforme Abildung von Gebieten der Randen von beschranken Drehung sind, Ann. Acad. Sci. Fennicae, Ser. A, no. 9 (1931), pp. 78.

9. C. J. Titus, The image of the boundary under a local homeomorphism, Lectures on Functions of a Complex Variable, Univ. of Michigan Press (1955), 433-35.

10. - The image of the boundary under an interior mapping, to appear 1960-61.

11. T. Umezawa, On the theory of univalent functions, Tohoku Math. J. 7 (1995), 212-228.

12. Hassler Whitney, Differentiable Manifolds, Annals of Math. 37 (1936),

13. - On regular closed curves in the plane, Compositio Math. 4 (1937), 276-284.

UNIVERSITY OF MICHIGAN 


\section{PACIFIC JOURNAL OF MATHEMATICS}

\section{EDITORS}

\author{
David Gilbarg \\ Stanford University \\ Stanford, California \\ F. H. Brownell \\ University of Washington \\ Seattle 5 , Washington
}

\author{
A. L. Whiteman \\ University of Southern California \\ Los Angeles 7, California \\ L. J. PAIGE \\ University of California \\ Los Angeles 24, California
}

\section{ASSOCIATE EDITORS}

\author{
E. F. BECKENBACH \\ T. M. CHERRY \\ D. DERRY
}
E. HEWITT
A. HORN
L. NACHBIN
M. OHTSUKA
H. L. ROYDEN
M. M. SCHIFFER

E. SPANIER

E. G. STRAUS

F. WOLF

\section{SUPPORTING INSTITUTIONS}

\author{
UNIVERSITY OF BRITISH COLUMBIA \\ CALIFORNIA INSTITUTE OF TECHNOLOGY \\ UNIVERSITY OF CALIFORNIA \\ MONTANA STATE UNIVERSITY \\ UNIVERSITY OF NEVADA \\ NEW MEXICO STATE UNIVERSITY \\ OREGON STATE COLLEGE \\ UNIVERSITY OF OREGON \\ OSAKA UNIVERSITY \\ UNIVERSITY OF SOUTHERN CALIFORNIA
}

\author{
STANFORD UNIVERSITY \\ UNIVERSITY OF TOKYO \\ UNIVERSITY OF UTAH \\ WASHINGTON STATE COLLEGE \\ UNIVERSITY OF WASHINGTON

AMERICAN MATHEMATICAL SOCIETY
CALIFORNIA RESEARCH CORPORATION
HUGHES AIRCRAFT COMPANY
SPACE TECHNOLOGY LABORATORIES
NAVAL ORDNANCE TEST STATION

Mathematical papers intended for publication in the Pacific Journal of Mathematics should be typewritten (double spaced), and the author should keep a complete copy. Manuscripts may be sent to any one of the four editors. All other communications to the editors should be addressed to the managing editor, L. J. Paige at the University of California, Los Angeles 24, California. ..

50 reprints per author of each article are furnished free of charge; additional copies may be obtained at cost in multiples of 50 .

The Pacific Journal of Mathematics is published quarterly, in March, June, September, and December. The price per volume (4 numbers) is $\$ 12.00$; single issues, $\$ 3.50$. Back numbers are available. Special price to individual faculty members of supporting institutions and to individual members of the American Mathematical Society: $\$ 4.00$ per volume; single issues, $\$ 1.25$.

Subscriptions, orders for back numbers, and changes of address should be sent to Pacific Journal of Mathematics, 2120 Oxford Street, Berkeley 4, California.

Printed at Kokusai Bunken Insatsusha (International Academic Printing Co., Ltd.), No. $6_{4}$ 2-chome, Fujimi-cho, Chiyoda-ku, Tokyo, Japan.

PUBLISHED BY PACIFIC JOURNAL OF MATHEMATICS, A NON-PROFIT CORPORATION

The Supporting Institutions listed above contribute to the cost of publication of this Journăl, but they are not owners or publishers and have no responsibility for its content or policies. 


\section{Pacific Journal of Mathematics}

\section{Vol. 10, No. $3 \quad$ November, 1960}

Glen Earl Baxter, An analytic problem whose solution follows from a simple

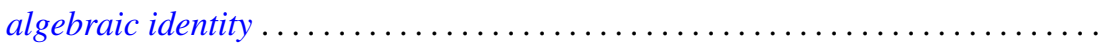

Leonard D. Berkovitz and Melvin Dresher, A multimove infinite game with linear payoff. .

Earl Robert Berkson, Sequel to a paper of A. E. Taylor ......................

Gerald Berman and Robert Jerome Silverman, Embedding of algebraic systems.... 767

Peter Crawley, Lattices whose congruences form a boolean algebra . . . . . ...... 777

Robert E. Edwards, Integral bases in inductive limit spaces . . . . . . . . . . . . . . .

Daniel T. Finkbeiner, II, Irreducible congruence relations on lattices . . . . . . . . . .

William James Firey, Isoperimetric ratios of Reuleaux polygons . . . . . . . . . . . 787

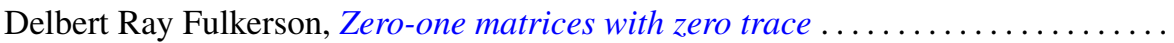

Leon W. Green, A sphere characterization related to Blaschke's conjecture........

Israel (Yitzchak) Nathan Herstein and Erwin Kleinfeld, Lie mappings in

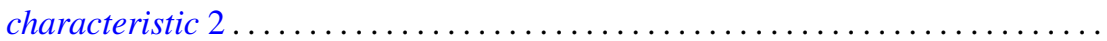

Charles Ray Hobby, A characteristic subgroup of a $p$-group .................

R. K. Juberg, On the Dirichlet problem for certain higher order parabolic

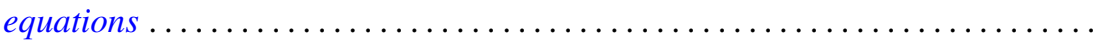

Melvin Katz, Infinitely repeatable games ......................

Emma Lehmer, On Jacobi functions . . . . . . . . . . . . . . . . . . . . . . . . .

D. H. Lehmer, Power character matrices

Henry B. Mann, A refinement of the fundamental theorem on the density of the sum of two sets of integers.

Marvin David Marcus and Roy Westwick, Linear maps on skew symmetric matrices: the invariance of elementary symmetric functions . .

Richard Dean Mayer and Richard Scott Pierce, Boolean algebras with ordered

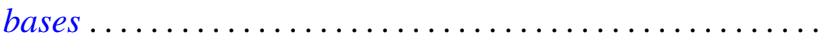

Trevor James McMinn, On the line segments of a convex surface in $E_{3} \ldots$

Frank Albert Raymond, The end point compactification of manifolds ..

Edgar Reich and S. E. Warschawski, On canonical conformal maps of regions of arbitrary connectivity

Marvin Rosenblum, The absolute continuity of Toeplitz's matrices...

Lee Albert Rubel, Maximal means and Tauberian theorems . .

Helmut Heinrich Schaefer, Some spectral properties of positive linear operators

Jeremiah Milton Stark, Minimum problems in the theory of pseudo-conformal transformations and their application to estimation of the curvature of the invariant metric.

Robert Steinberg, The simplicity of certain groups ...

Hisahiro Tamano, On paracompactness. .

Angus E. Taylor, Mittag-Leffler expansions and spectral theory .

Marion Franklin Tinsley, Permanents of cyclic matrices ...... . 